\title{
EXCESS LINEAR SERIES ON AN ALGEBRAIC CURVE ${ }^{1}$
}

\author{
WILLIAM FULTON, JOE HARRIS AND ROBERT LAZARSFELD
}

\begin{abstract}
We prove that the dimensions of the varieties $W_{d}^{r}$ of complete linear series of degree $d$ and dimension at least $r$ on a curve satisfy the inequalities $\operatorname{dim} W_{d-1}^{r} \geq \operatorname{dim} W_{d}^{r}-(r-1)$. In particular, a curve with $\infty^{2} g_{d}^{1}$ 's must have a $g_{d-1}^{1}$.
\end{abstract}

In [4] it was seen that new results in the theory of linear systems on an algebraic curve could be obtained by using properties of ample vector bundles. The purpose of the present note is to make some further observations along the same lines. Specifically, for any curve $C$ of genus $g$, we denote by $W_{d}^{r}=W_{d}^{r}(C)$ the variety of complete linear systems of degree $d$ and dimension at least $r$ on $C$. Our goal is to prove

THEOREM 1. For any $C, \operatorname{dim} W_{d-1}^{r} \geq \operatorname{dim} W_{d}^{r}-(r+1)$.

This theorem has a number of immediate consequences. For example, observing that, by Riemann-Roch,

$$
W_{d}^{r} \cong W_{2 g-2-d}^{g-d+r-1}, \quad W_{d+1}^{r+1} \cong W_{2 g-2-d-1}^{g-d+r-1},
$$

and applying Theorem 1, we have

$$
\text { COROLlaRY 2. } \operatorname{dim} W_{d+1}^{r+1} \geq \operatorname{dim} W_{d}^{r}-(g-d+r) .
$$

If we now combine Theorem 1 and Corollary 2, we have

COROLlaRY $3 . \operatorname{dim} W_{d}^{r+1} \geq \operatorname{dim} W_{d}^{r}-(g-d+2 r+2)$.

One special case of Theorem 1 is the statement that

$$
\operatorname{dim} W_{d-1}^{1} \geq \operatorname{dim} W_{d}^{1}-2 .
$$

In particular, if a curve $C$ has a 2-dimensional family of $g_{d}^{1}$ 's, it must have a $g_{d-1}^{1} .^{2}$ This is in response to a question of Arthur Ogus.

REMARK. As will be apparent from the proof, Theorem 1 may be strengthened to say that if we choose any basepoint $p_{0} \in C$ and correspondingly view $W_{d-1}^{r}$ as a subvariety of $W_{d}^{r}$, and if $\Sigma$ is any irreducible component of $W_{d}^{r}$, then

$$
\operatorname{dim}\left(\Sigma \cap W_{d-1}^{r}\right) \geq \operatorname{dim} \Sigma-(r+1) .
$$

The other statements can be similarly sharpened.

\footnotetext{
Received by the editors October 31, 1983.
}

1980 Mathematics Subject Classification. Primary 14C20.

${ }^{1}$ Research partially supported by the National Science Foundation.

${ }^{2}$ The referee points out that this last statement has also been proved by M. Coppens (Some sufficient conditions for the gonality of a smooth curve, J. Pure Appl. Algebra 30 (1983), 5-21; Theorem 11). 
REMARK. On a general curve $C$ of genus $g$, we have

$$
\operatorname{dim} W_{d}^{r}=\rho=g-(r+1)(g-d+r)
$$

and so equality holds in the theorem and corollaries when $C$ is general. Thus these results go beyond the standard Brill-Noether theory exactly in case $\operatorname{dim} W_{d}^{r}>\rho$, that is, $C$ has excess linear systems.

The proof of Theorem 1 is based on the easy

LEMMA 4. Let $E, F$ and $F^{\prime}$ be vector bundles of ranks $m, n$ and $n+1$ on a projective variety $X, \pi: F^{\prime} \rightarrow F$ a surjection with kernel $L, \sigma: E \rightarrow F^{\prime}$ any bundle map, and $\Sigma^{\prime}$ (resp., $\left.\Sigma\right)$ the locus where $\sigma(r e s p . \pi \circ \sigma)$ has rank $k$ or less. Assume that $E^{*} \otimes L=\operatorname{Hom}(E, L)$ is ample. Then

$$
\operatorname{dim} \Sigma^{\prime} \geq \operatorname{dim} \Sigma-(m-k) .
$$

PROOF. First, intersecting with a general subvariety $H$ of codimension $\operatorname{dim} \Sigma-(m-k)$ in $X$, it is sufficient to show

$$
\operatorname{dim}\left(\Sigma^{\prime} \cap H\right) \geq \operatorname{dim}(\Sigma \cap H)-(m-k)=0 .
$$

That is, we may assume $\operatorname{dim} \Sigma=m-k$, and then show that $\Sigma^{\prime}$ is not empty. Now if $\operatorname{rank}(\pi \circ \sigma)_{x} \leq k-1$ for any $x \in \Sigma$, then $\operatorname{rank} \sigma_{x} \leq k$ and we are done. We therefore assume $\operatorname{rank}(\pi \circ \sigma)=k$ throughout $\Sigma$, i.e. that $G=\operatorname{Ker}(\pi \circ \sigma)$ is a $\operatorname{rank} m-k$ sub-vector-bundle of $E$. We then have an induced map $\tilde{\sigma}: G \rightarrow L$, and clearly $\Sigma^{\prime}$ is just the zero-locus in $\Sigma$ of this map. But $\operatorname{Hom}(G, L)=G^{*} \otimes L$ is a quotient bundle of $E^{*} \otimes L$, and so, by hypothesis, ample. By the arguments of $[\mathbf{2}, \mathbf{4}$ or $\mathbf{1}$, Chapter $7, \S 1]$, a global section of an ample vector bundle whose rank is less than or equal to the dimension of its base must have a zero.

To deduce Theorem 1 from the Lemma, we use the by-now standard determinantal description of $W_{d}^{r}$ (cf. [4]): fix $e \geq 2 g-d-1$ and denote by $\mathcal{L}$ the Poincaré line bundle on $C \times \operatorname{Pic}^{e}(C)$ (that is, the bundle whose restriction to each fiber $C \times\{L\}$ is isomorphic to $L)$, normalized so that $\left.\mathcal{L}\right|_{\left\{p_{0}\right\} \times \operatorname{Pic}^{e}(C)}$ is trivial; take $E=\left(\pi_{2}\right)_{*} \mathcal{L}$ (that is, the bundle whose fiber over $L \in \mathrm{Pic}^{e}(C)$ is $H^{0}(C, L)$ ) and, for distinct points $p_{0}, \ldots, p_{e-d} \in C$,

$$
F=\bigoplus_{i=1}^{e-d} L_{p_{i}}, \quad F^{\prime}=\bigoplus_{i=0}^{e-d} L_{p_{i}},
$$

where $L_{p}=\left.\mathcal{L}\right|_{\{p\} \times \operatorname{Pic}^{e}(C)}$. Then $W_{d}^{r}$ (resp., $\left.W_{d-1}^{r}\right)$ is, up to translation, the locus where the natural evaluation map $E \rightarrow F$ (resp., $E \rightarrow F^{\prime}$ ) has rank $e-g-r$ or less. Since, finally, the bundle $E^{*}$ is ample (cf. $[\mathbf{1}$ or $\mathbf{4}, \S 2]$ ) we may apply Lemma 4 to conclude Theorem 1 .

REMARK. Given a map $\phi$ from a bundle $E$ to a bundle $F$ with filtration $0 \subset F_{1} \subset \cdots \subset F_{k}=F$ such that $E^{*} \otimes F_{i} / F_{i-1}$ is ample for each $i$, we may ask whether the codimensions of the various degeneracy loci associated to $\phi$ (as in $[3])$ in one another are necessarily no greater than expected. We do not know the answer to this, except that it is not always the case. For example, in the setting of Lemma 4 above, if $\Sigma^{\prime \prime}$ is the locus where $\pi \circ \sigma$ has rank $k-1$ or less, it is not necessarily true that

$$
\operatorname{dim} \Sigma^{\prime \prime} \geq \operatorname{dim} \Sigma^{\prime}-(n-k+1)
$$

although this does follow in the special case of the bundle map above by Serre duality, as in Corollary 2. 


\section{REFERENCES}

1. E. Arbarello, M. Cornalba, P. Griffiths and J. Harris, Geometry of algebraic curves, SpringerVerlag, 1984.

2. S. Bloch and D. Gieseker, The positivity of the Chern classes of an ample vector bundle, Invent. Math. 12 (1971), 112-117.

3. W. Fulton, Intersection theory, Springer-Verlag, 1983.

4. W. Fulton and R. Lazarsfeld, On the connectedness of degeneracy loci and special divisors, Acta Math. 146 (1981), 271-283.

Department of Mathematics, Brown University, Providence, Rhode Island 02912 (Current address of William Fulton and Joe Harris)

Department of Mathematics, Harvard University, Cambridge, MassachuSETTS 02138

Current address (R. Lazarsfeld): Department of Mathematics, University of California, Los Angeles, California 90024 